\title{
Quasi-Black Mask for Low-Cost LCDs by Patterned Alignment Films Formed by an Electro-Spray Deposition Method**
}

\author{
Yukihiro KUDOH $^{\dagger \text { a) }}$, Yuta UCHIDA ${ }^{\dagger \dagger *}$, and Taiju TAKAHASHI ${ }^{\dagger}$, Nonmembers
}

\begin{abstract}
SUMMARY A black mask (BM) is a layer used to improve the display quality by suppressing light leakage. In general, the BM is formed by a photolithography process. In this study, a novel technique for the fabrication of a quasi-black mask (q-BM) is proposed; the q-BM was composed of vertical and hybrid orientation areas, patterned by a separation coating technique using an electro-spray deposition method. Using our technique, the q-BM can be formed easily without the additional masks used for the BM.

key words: electro-spray deposition, liquid crystal display, selective coating, black-mask
\end{abstract}

\section{Introduction}

The electro-spray deposition (ESD) method is a technique for forming a thin film using an electric field and charge, first proposed by Morozov et al. [1]-[9]. In general, in the ESD technique, a liquid solution droplet on the capillary top is charged by electric charges because of the application of a very strong electric field between the substrate and the capillary. The droplet that is removed from the capillary is spotted of again and again by the Coulomb force applied to the electric charge. During this process, tiny droplets fly to a metal plate connected to the electrical ground, depositing the materials on the substrate placed on the metal plate.

At present, the fabrication of patterned films by the ESD method can be performed using either the mechanicalmask [10]-[12] or patterned electrode techniques. In the latter, misty tiny droplets can be selectively deposited on the glass substrate surface with electrode patterns under optimized ESD conditions [13].

In previous studies, our research group has investigated the application of the ESD method for forming liquid crystal (LC) alignment films [14]-[16]. When the ESD method was used to form an alignment film comprising numerous small domains with two types of alignment materials for the horizontal and vertical alignment, the pretilt angle was controlled using simple equipment in the atmosphere [15]. Fur-

\footnotetext{
Manuscript received February 29, 2016.

Manuscript revised June 7, 2016.

$\dagger$ The authors are with the Department of Information and Communications Engineering, Kogakuin University, Hachioji-shi, 1920015 Japan.

${ }^{\dagger \dagger}$ The author is with the Graduate School of Engineering, Kogakuin University, Hachioji-shi, 192-0015 Japan.

*Presently, the author has completed a master's degree and is working in an electronics company.

**This paper was presented at the 22nd International Display Workshops.

a)E-mail: kudoh@cc.kogakuin.ac.jp

DOI: $10.1587 /$ transele.E99.C.1244
}

thermore, the selective coating method was proposed for the system with two types of alignment materials, for example the horizontal and vertical alignment materials [17]. This is achieved by using a combination of the ESD and conventional spin-coating methods. When the ESD method was applied to maskless patterning by using electrodes on the substrate, materials were deposited on the electrodes spontaneously because of the Coulomb force, leading to highly efficient use of the material.

\section{Quasi-Black Mask (q-BM)}

A black mask (BM) [18], [19] is a layer used to improve the display quality by suppressing light leakage. In general, the BM is formed by a photolithography process. In this study, we propose a novel technique to fabricate a quasiblack mask (q-BM) by using ESD. We focus our study on the passive-driven display with a segment electrode, such as with a seven-segment pattern. Passive-driven displays are used as low-cost displays in applications such as operation panels of electronic devices. The passive driven display has pixel and extraction electrodes for the application of voltage to the pixel electrode. The use of the BM such displays improves the display quality by preventing the transmission of light in the areas outside the pixel electrodes. However, the fabrication of BM can be expensive, increasing the cost of high-quality LCs. By applying the ESD method for the production of a pseudo-black mask in the orientation film fabrication process, we aim to improve the display quality without raising the display cost.

The q-BM can be fabricated easily without masks. Figure 1 shows the example of LC alignments in an LC cell with the q-BM obtained using a separation coating technique. The patterned alignment layer is prepared as follows: a uniform vertical alignment film is formed on a glass substrate with indium doped tin oxide (ITO) pattern using conventional methods such as spin-coating, and the horizontal alignment material is then deposited on the electrode using the separation coating technique of the ESD method. To assemble these substrates, the LC orientation in the cell is obtained as shown in Fig. 1. A horizontal orientation is realized only on the electrode, and the other area of the LC shows a vertical orientation and functioned as a BM. Additionally, a black state is also realized on a region of extraction electrodes due to an optical extinction condition with a hybrid orientation. Thus, our q-BM can be used to block the transmission of the light outside of the pixel area, leading to 


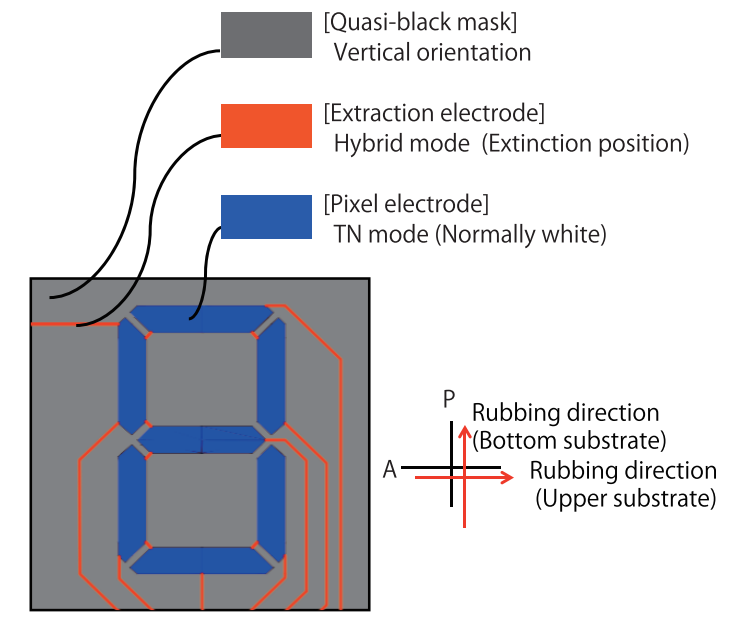

Fig. 1 LC orientations in the seven-segment cell with quasi-black mask [20], [21].

expected improvements in display quality.

\section{Experimental Procedure}

In this experiment, a seven-segment LCD with the q-BM was used to demonstrated the performance of our new fabrication method.

Electrode patterns (Figs. 2 (a) and (b)) for the sevensegment LCD were formed on glass substrates $(25 \times 20 \times$ $1.1 \mathrm{~mm}$ ) using the photolithography process. Then, the vertical alignment material, SE-1211 (Nissan Chemical Industries) $4 \mathrm{wt} \%$, was spin-coated and a heat treatment at $220^{\circ} \mathrm{C}$ for $1 \mathrm{~h}$ was performed. Next, the horizontal alignment material, PI-C (Nissan Chemical Industries) 4 wt\%, was deposited selectively on the electrode patterns by the ESD method, as shown in Fig. 3. For the ESD treatment, an alignment material solution diluted 10 -fold with a solvent mixture of acetonitrile and tetrahydrofuran (4/6 wt/wt) was used. We used a capillary with an internal diameter of $50 \mu \mathrm{m}$ and applied a voltage of $5 \mathrm{kV}$ to the capillary.

The sample cells were fabricated using two different ESD instruments for the separation coating. The first one is generally used ESD instrument: a substrate holder fabricated of the insulator material faces the capillary tip as shown in Fig. 3(a). The second instrument is a specially improved instrument with the auxiliary spherical electrode facing the capillary tip as shown in Fig. 3 (b). A metal sphere with a diameter of $3 \mathrm{~cm}$ was used as an auxiliary electrode to catch the large droplets and prevent the depositing of these large size droplets on the substrates. This auxiliary electrode was placed just above the capillary tip, and the glass substrate was placed on the concentric circles centered on the capillary tip. Furthermore, the substrate was set up parallel to the tangent of the circle. Here, the substrate angle $\phi$ was defined by the horizontal line as zero. Sample cells were fabricated with different substrate angle $\phi(\phi=15,25$, and $35^{\circ}$ ).

After spraying with PI-C solution to improve the film

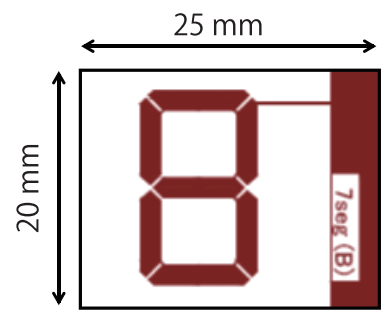

(a) Common electrode.

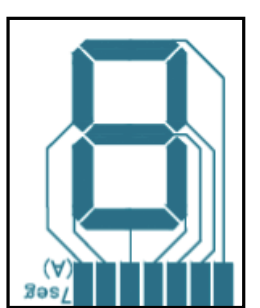

(b) Pixel electrodes.

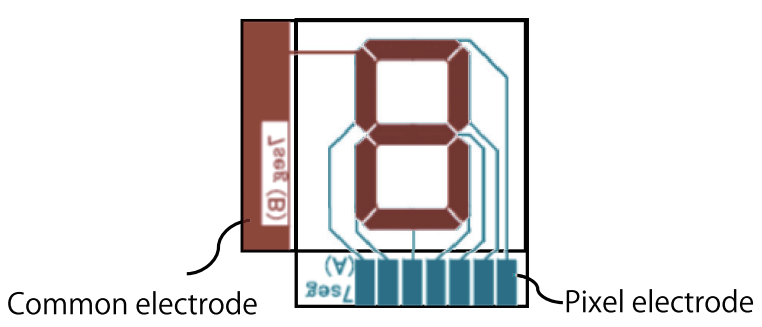

(c) Assembled cell.

Fig. 2 Schematic of sample LC cell [20], [21].

adhesion, $10 \mu \mathrm{l}$ of gamma-butyrolactone was sprayed over the substrate surface by ESD. This was followed by heat treatment at $220^{\circ} \mathrm{C}$ for $1 \mathrm{~h}$ and a rubbing treatment. Finally, the empty cells with a thickness of $6 \mu \mathrm{m}$ were assembled as shown in Fig. 2 (c), and the nematic liquid crystal 5CB (Merck) was injected into the cell with the isotropic phase and cooled down to room temperature.

\section{Results and Discussion}

As mentioned above, in the ideal LC mode, the LC director configuration on the segment electrodes was in the $90^{\circ}$ $\mathrm{TN}$ state, wheres that on the extraction electrodes was in the hybrid state with optical extinction position, and vertical alignment was obtained in the other area as shown in Fig. 1.

Figure 4 shows images of the sample cell fabricated using the general ESD instrument (Fig. 3 (a)). Horizontal LC oriented areas were observed not only on the pixel electrode but also on the other areas in the upper right side of the sample cell. This is attributed to the instability of the Taylor cone at the capillary tip. The Taylor cone is formed due to the balance between the surface tension of the solution and the Coulomb force, and the electric field concentration due to the ITO electrode on the glass substrate disrupts this balance, leading to the spraying of large droplets. On the other hand, relatively favorable patterning following our design was realized on the left side of the sample cell; the bright state was located in the segment pattern area, and the dark state due to the vertical alignment was observed. This shows that it is important to decrease the size of the droplets by improving the stability of the Taylor cone.

Figure 5 shows the images of sample cells fabricated with the improved ESD instrument using the crossed Nicols. The cells were fabricated with different substrate angle $\phi$ in the ESD process. Using the improved ESD instrument, in 


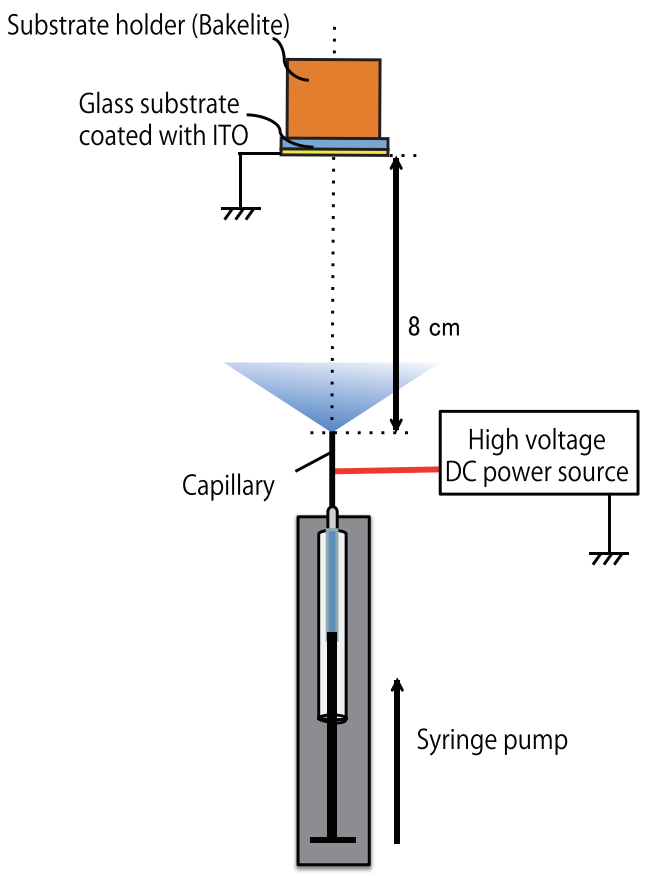

(a) General implement.

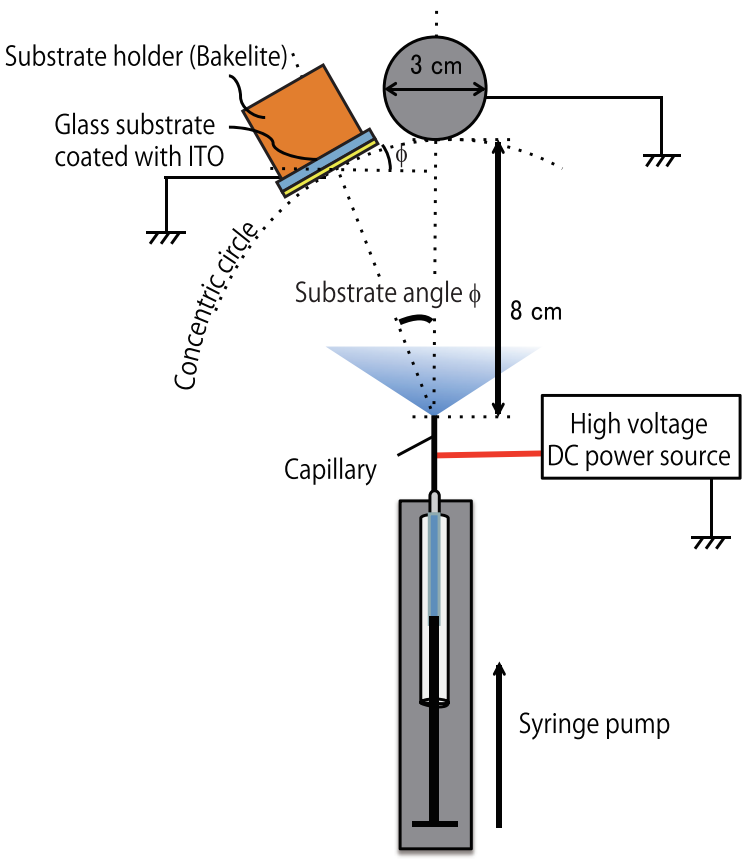

(a) Improved implement.

Fig. 3 Schematic of our ESD instrument [20], [21].

all conditions, better alignment was obtained that that of the sample LC cell fabricated by the conventional instrument. We attribute this to the stabilization of the Taylor cone by the auxiliary electrode. Additionally, large droplets fly to the region between the substrate and auxiliary electrode because the size of the available substrate is increased by the auxiliary electrodes.

The bright state was observed in the segment pattern area due to the optical rotation by the $90^{\circ}$ twisted nematic

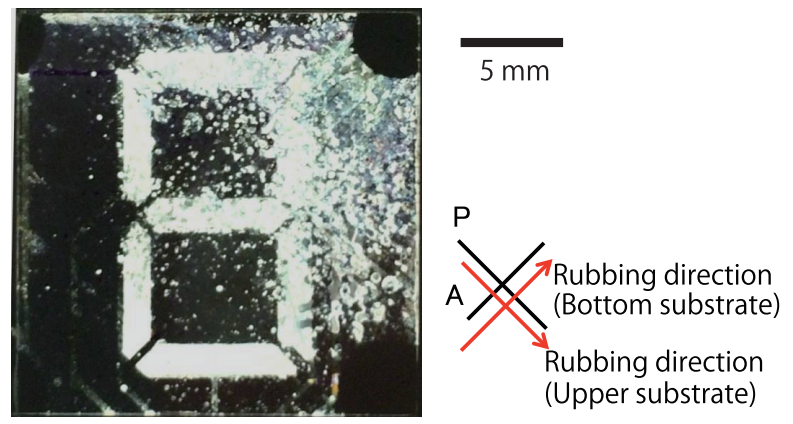

Fig. 4 A photo of the sample cell with the quasi-black mask fabricated by the general ESD instrument.

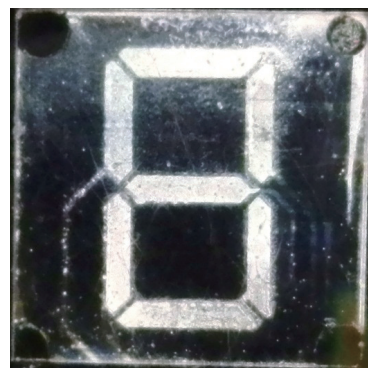

(a) $\phi=15^{\circ}$

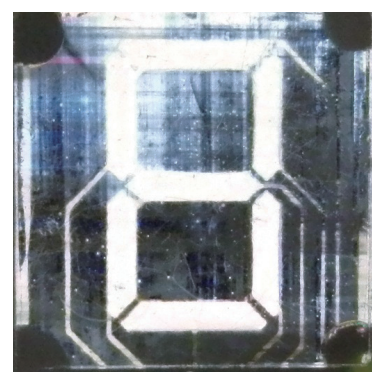

(c) $\phi=35^{\circ}$

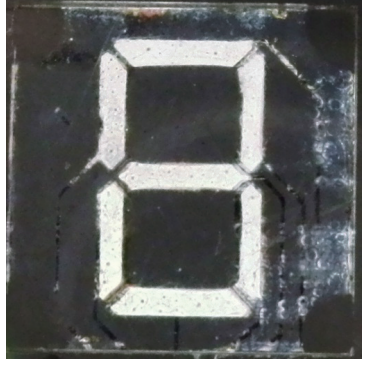

(b) $\phi=25^{\circ}$

$5 \mathrm{~mm}$

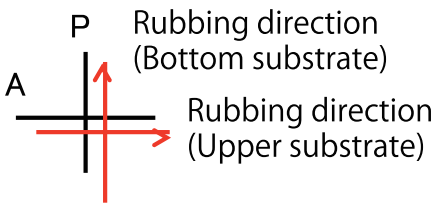

Fig.5 Photos of the sample cells with the quasi-black mask fabricated by improved ESD instrument [20], [21].

(TN) configuration. In contrast, the dark state due to the vertical alignment was observed in the no electrode area. Furthermore, the dark state was also obtained at the regions of extraction electrodes with the hybrid alignment. However, non-uniform LC orientations were observed in those areas. This was attributed to the small amount of the horizontal alignment material deposited on the no electrode parts. The substrate angle $\phi$ influenced the amount of the deposited alignment material. When the auxiliary electrode and the substrate were close to each other, many tiny droplets with horizontal alignment material were deposited on the no electrode regions on the substrate.

In the sample cell fabricated with $\phi=15^{\circ}$, some bright dots were observed. This was attributed to the deposition of the horizontal alignment material on the area without the electrodes because the auxiliary electrode was very close to the substrate. 


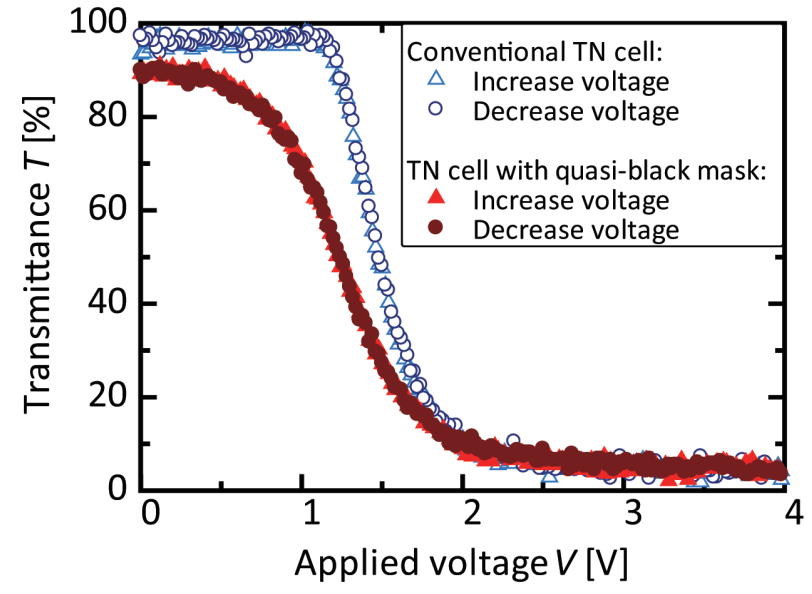

Fig. 6 T-V characteristics of the LC cells.

Additionally, the incompletely dark state with scraped texture generated by rubbing treatment was observed on the area without the electrodes in the sample cell for $\phi=35^{\circ}$. This was due to the horizontal alignment droplets drifting in the peripheral part of the spray reaching the substrate and their deposition on the area without the electrode. Additionally, the bright state was observed in some parts of the extraction electrode patterns in this sample. This was attributed to the same reason; if the horizontal alignment material was deposited on the extraction electrode, the director configuration in these sections was not that of a hybrid orientation but rather of a $90^{\circ} \mathrm{TN}$ orientation.

Accordingly, the substrate angle of $\phi=25^{\circ}$ was found to be optimal in this experiment. However, it was necessary to improve the uniformity of the precision of the selective coating by the ESD method.

Figure 6 shows the transmittance vs. applied voltage $\left(\mathrm{T}-\mathrm{V}\right.$ ) behavior of the general $90^{\circ} \mathrm{TN}$ cell and the sample LC cell with $\phi=25^{\circ}$. Here, the T-V characteristics were measured under the polarized microscope (Olympus BX51) with an $\times 20$ of objective lens, and a photodiode was mounted to the eyepiece portion. The white light source of the microscope was used.

Unfortunately, compared to the general TN cell, a slightly lower contrast ratio and gentler threshold characteristics were obtained in the LC cell with q-BM. This was most likely due to the occurrence of optical scattering caused by a rough alignment film surface. Additionally, it was considered that higher pretilt angle required for the $\mathrm{TN}$ mode was induced on the electrode in the q-BM cell. Because the horizontal alignment film was formed over the vertical alignment film, as a result, the vertical alignment film might be dissolved out by spraying the horizontal alignment material, or side chains of the vertical alignment material might affect to the surface on the horizontal alignment film. Fortunately, no hysteresis characteristic was observed in the T-V curves. This result shows that the alignment film formed by the ESD has an enough anchoring strength and a good stability.

\section{Conclusions}

A novel q-BM structure was proposed and fabricated by a selective coating technique using the ESD method. Using our technique, the q-BM for LCDs can be formed easily without any additional photo masks typically used for the formation of the BM layer. Furthermore, a prototype sevensegment LCD with the q-BM was successfully fabricated according to our design. However, non-uniform LC textures were observed in pixel areas due to the small amount of the horizontal alignment material deposited on the no electrode regions of the substrate. Thus, for the further development of this technique, it is necessary to improve the uniformity of the alignment film and the control of the thickness of the selective coating deposited by the ESD method.

\section{Acknowledgments}

We would like to thank Merck Co., Ltd. for supplying the LC materials. We also thank Nissan Chemical Industries Ltd. for supplying the polyimide materials.

\section{References}

[1] V.N. Morozov and T.Y. Morozova, "Electrospray deposition as a method to fabricate functionally active protein films," Anal. Chem., vol.71, no.7, pp.1415-1420, 1999.

[2] V.N. Morozov and T.Y. Morozova, "Electrospray deposition as a method for mass fabrication of mono- and multicomponent microarrays of biological and biologically active substances," Anal. Chem., vol.71, no.15, pp.3110-3117, 1999.

[3] B. Lee, J. Kim, K. Ishimoto, Y. Yamagata, A. Tanioka, and T. Nagamune, "Fabrication of protein microarrays for immunoassay using the electrospray deposition (ESD) method," Journal of Chemical Engineering of Japan, vol.36, no.11, pp.1370-1375, 2003.

[4] K. Morota, H. Matsumoto, T. Mizukoshi, Y. Konosu, M. Minagawa, A. Tanioka, Y. Yamagata, and K. Inoue, "Poly(ethylene oxide) thin films produced by electrospray deposition: Morphology control and additive effects of alcohols on nanostructure," Journal of Colloid and Interface Science, vol.279, no.2, pp.484-492, 2004.

[5] H. Matsumoto, T. Mizukoshi, K. Nitta, M. Minagawa, A. Tanioka, and Y. Yamagata, "Organic/inorganic hybrid nano-microstructured coatings on insulated substrates by electrospray deposition," Journal of Colloid and Interface Science, vol.286, no.1, pp.414-416, 2005.

[6] I.B. Rietveld, K. Kobayashi, H. Yamada, and K. Matsushige, "Electrospray deposition, model, and experiment: Toward general control of film morphology," Journal of Physical Chemistry B, vol.110, no.46, pp.23351-23364, 2006.

[7] I.B. Rietveld, K. Kobayashi, H. Yamada, and K. Matsushige, "Morphology control of poly(vinylidene fluoride) thin film made with electrospray," Journal of Colloid and Interface Science, vol.298, no.2, pp.639-651, 2006.

[8] A. Jaworek, "Electrospray droplet sources for thin film deposition," Journal of Materials Science, vol.42, no.1, pp.266-297, 2007.

[9] B.G. Prajapati and M. Patel, "A technology update: Electro spray technology," International Journal of Pharmaceutical Sciences Review and Research, vol.1, no.1, pp.12-13, 2010.

[10] Y. Yamagata, "Micro/nano patterning by electrospray deposition method," Seikei Kakou [in Japanese], vol.17, no.6, pp.364-367, 2005.

[11] J.-W. Kim, Y. Yamagata, B.J. Kim, and T. Higuchi, "Direct and dry micro-patterning of nano-particles by electrospray deposition 
through a micro-stencil mask," Journal of Micromechanics and Microengineering, vol.19, no.2, 025021, 2009.

[12] J. Ju, Y. Yamagata, and T. Higuchi, "Thin-film fabrication method for organic light-emitting diodes using electrospray deposition," Advanced Materials, vol.21, no.43, pp.4343-4347, 2009.

[13] S. Khan, Y.H. Doh, A. Khan, A. Rahman, K.H. Choi, and D.S. Kim, "Direct patterning and electrospray deposition through EHD for fabrication of printed thin film transistors," Current Applied Physics, vol.11, no.1, Supplement, pp.S271-S279, 2011.

[14] Y. Kudoh, S. Saito, and T. Takahashi, "Application of an electro spray deposition (ESD) method to control the pre-tilt angle in a nematic LC cell," Molecular Crystals and Liquid Crystals, vol.546, no.1, pp.156-162, 2011.

[15] Y. Kudoh, Y. Uchida, and T. Takahashi, "Control of the pretilt angle for liquid crystal alignment using novel electrospray deposition methods with two capillaries," Journal of the Society for Information Display, vol.22, no.7, pp.357-363, 2014.

[16] Y. Kudoh, Y. Uchida, and T. Takahashi, "Control of pre-tilt angle and evaluation of azimuthal anchoring strength considering the pre-tilt angle on the alignment film formed by the ESD method," Molecular Crystals and Liquid Crystals, vol.610, no.1, pp.210-216, 2015.

[17] Y. Uchida, Y. Kudoh, and T. Takahashi, "Separation coating method for alignment materials by using the ESD technique on the glass substrate with patterned ITO electrodes," Proc. 14th International Meeting on Information Display, p.489, 2014.

[18] P.M. Harrison, J. Wendland, and M. Henry, "49.2: Innovative laser patterning of black matrix for LCD manufacture," SID Symposium Digest of Technical Papers, vol.39, no.1, pp.736-739, 2008.

[19] B.D. Lee, Y.-H. Cho, M.H. Oh, S.Y. Lee, S.Y. Lee, J.H. Lee, and D.S. Zang, "Characteristics of contrast of active-matrix organic light-emitting diodes containing a black matrix and antireflection layers," Materials Chemistry and Physics, vol.112, no.3, pp.734-737, 2008.

[20] Y. Kudoh, Y. Uchida, and T. Takahashi, "Fabrication of quasi-black mask for LCDs by selective coating technique using an electro-spray deposition method," Proc. 22nd International Display Workshops, pp.473-476, 2015

[21] Y. Kudoh, Studies on novel techniques for control of the liquid crystal orientation by using electro-spray deposition and electrospinning methodsiquid crystal orientation by using electro-spray deposition and electrospinning methods, $\mathrm{PhD}$ thesis, Graduate School of Engineering, Kogakuin University, 2015.

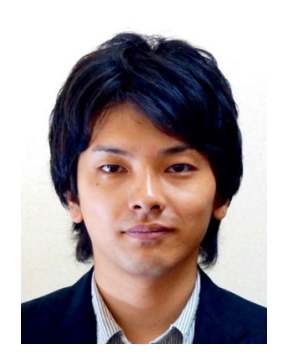

Yukihiro Kudoh received a B.S. degree in Information and Communications Engineering from Kogakuin University in 2010 and an M.S. and Ph.D. in Electrical Engineering and Electronics from the same university in 2012 and 2015, respectively. Currently, he is an Assistant Professor at the Department of Information and Communications Engineering at the Kogakuin University.

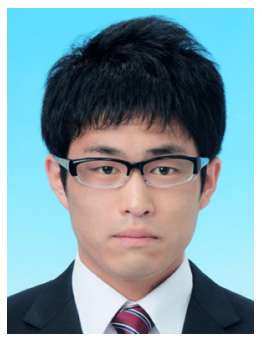

Yuta Uchida received a B.S. degree in Information and Communications Engineering from Kogakuin University in 2012 and an M.S. degree in Electrical Engineering and Electronics from the same university in 2015. Presently, he is working in a company as an Electronics Engineer.

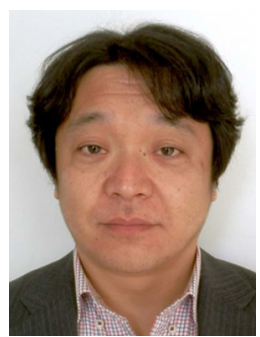

Taiju Takahashi received B.S. and M.S. degrees in Electronic Engineering and Electrical Engineering in 1989 and 1991, respectively, from Kogakuin University. After completion of his M.S. degree, he used to be a researcher at a company until 1995. After that, he received a Ph.D. from Nagaoka University of Technology in 1998. Thereafter, he worked as a postdoctoral fellow at the Liquid Crystal Institute of Science University of Tokyo in Yamaguchi until 2000. Currently, he is a Professor at the Department of Information and Communications Engineering at the Kogakuin University. 